\title{
The construction of one unstable manifold for the dissipative Hénon mapping
}

\author{
Theo Valkering \\ Center for Theoretical Physics, Twente University of Technology, PO Box 217, 7500 AE \\ Enschede, The Netherlands
}

Received 17 April 1984, in final form 22 June 1984

\begin{abstract}
The unstable manifold of a saddle point of the Hénon mapping is constructed analytically via a contraction mapping, for a range of parameter values where the second fixed point is a stable node. One invariant piece of this manifold connects the saddle with the second fixed point. Rigorous error bounds are derived for the each step of the iterative procedure. It is demonstrated that an algebraic approximation with known accuracy can be given of the unstable manifold.
\end{abstract}

\section{Introduction}

The dissipative Hénon mapping (Hénon 1976) in the form discussed by Helleman (1983)

$$
H: \underset{y}{x} \rightarrow \begin{aligned}
& f(x)-B y \\
& x
\end{aligned}, \quad f(x)=2 C x+2 x^{2},
$$

has two fixed points, one at the origin $\mathrm{O}$ and a second one at a point $\mathrm{A}$. One invariant manifold, connecting both fixed points, is constructed for $0<B<1$ and such $C$ values that the origin is an attractor with real positive eigenvalues and that $A$ is a saddle in the right upper half plane. Every point on this manifold, except $A$, approaches the origin monotonically under repeated application of the mapping. A second invariant manifold is constructed joining up smoothly with the first one at A. All points of that second manifold move monotonically away from $A$ to infinity. Both manifolds together constitute the complete unstable manifold of $\mathrm{A}$.

The main idea of the construction of the first manifold is to obtain for a suitable initial curve $\Gamma_{b}$, connecting $\mathrm{O}$ and $\mathrm{A}$, a convergent sequence of curves $H^{n} \Gamma_{b}$, where $H$ denotes the Hénon mapping. The limiting curve, $\Gamma_{1}$, satisfies $\Gamma_{1}=H \Gamma_{1}$. To study the convergence these curves are represented as elements of a metric function space and correspondingly, a nonlinear operator representing $H$, is defined on this space. This operator is contractive on a subspace $\mathscr{S}$ and the solution of its fixed point equation yields $\Gamma_{1}$. The solution is constructed in the usual way, i.e. by repeatedly applying the operator to some arbitrary element in $\mathscr{F}$. With the aid of the contractive property the accuracy at each step is determined. The second invariant manifold, $\Gamma_{2}$, is constructed in a similar way. The contraction mapping principle was used to prove the existence of invariant manifolds locally (Nitecki 1971, Lanford 1983). Due to the above described restriction of the parameter values the contraction property can be formulated in such a way that the present global results are obtained. 


\section{A functional fixed point equation for the invariant manifold}

Functional equations are derived here for functions describing the invariant manifolds $\Gamma_{1}$ and $\Gamma_{2}$. Each equation is interpreted as a fixed point equation in a metric function space.

Let the fixed point A have coordinates $(A, A)$, with $A>0$, and consider a continuous invertible function $\gamma(y)$ defined on $0 \leqslant y<\infty$, with $\dagger$

$$
\gamma(0)=0, \quad \gamma(A)=A, \quad \gamma^{\prime}(y)>0,
$$

whose graph $\Gamma$ is to describe the invariant manifold. This means that any point $(\gamma(y), y)$ of $\Gamma$ is mapped by the Hénon mapping onto another point $\left(\gamma\left(y^{*}\right), y^{*}\right)$,

$$
\gamma\left(y^{*}\right)=f(\gamma(y))-B y, \quad y^{*}=\gamma(y) .
$$

The function $\gamma(y)$, considered as a mapping of $[0, \infty)$ onto itself $(\operatorname{cf}(2.2 b))$, has $[0, A]$ and $[A, \infty)$ as invariant intervals. Correspondingly $\Gamma$ is the union of two sets

$$
\Gamma_{1}=\{(\gamma(y), y) \mid 0 \leqslant y \leqslant A\}, \quad \Gamma_{2}=\{(\gamma(y), y) \mid A \leqslant y<\infty\},
$$

each of which is invariant under $H$. The functional equation for $\gamma$ is obtained by substituting $y=\gamma^{-1}\left(y^{*}\right)$ (cf $\left.(2.2 b)\right)$ into $(2.2 a)$, and omitting the asterisk one finds

$$
\gamma(y)=f(y)-B \gamma^{-1}(y) \text {. }
$$

This equation was proposed earlier (McMillan 1971) and used by several others (Bridges and Rowlands 1977, Tel 1982, Daido 1980). After substitution of

$$
\gamma(y) \equiv y+g(y), \quad \gamma^{-1}(y) \equiv y+\tilde{g}(y)
$$

into $(2.4 b)$, we obtain our final equation

$$
g(y)=h(y)-B \tilde{g}(y), \quad \text { with } h(y)=f(y)-B y-y .
$$

Provided that $\gamma^{\prime}=1+g^{\prime}>0, \tilde{g}$ is uniquely determined and $(2.6 a)$ is a fixed point equation written in shorthand

$$
g=T g \text {. }
$$

The construction of $\tilde{g}$ from $g$ is demonstrated in figure 1 , which shows that $\tilde{g}$, on the interval $[0, A]$, is determined completely by $g$ on $[0, A]$. The same holds for the interval $[A, \infty)$. Thus (2.6) may be interpreted as an equation for functions $g$ defined on each of the two intervals. Figure 1 also shows that

$$
g(0)=g(A)=0, \quad \tilde{g}(0)=\tilde{g}(A)=0
$$

and correspondingly for the domain $[0, A]$ the linear function space

$$
\mathscr{F}_{1} \equiv\left\{g_{1}(y) \mid g_{1} \in \mathscr{C}^{1}([0, A]), g_{1}(0)=g_{1}(A)=0\right\}
$$

is defined, which is complete with respect to the norm $\ddagger$ (Brown and Page 1970)

$$
\|g\| \equiv \sup _{0 \leqslant y \leqslant A}\left|g^{\prime}(y)\right|
$$

$\dagger$ A prime denotes differentiation.

$\ddagger$ The index 1 is omitted if it is clear that the restriction to the domain $[0, A]$ is meant. 

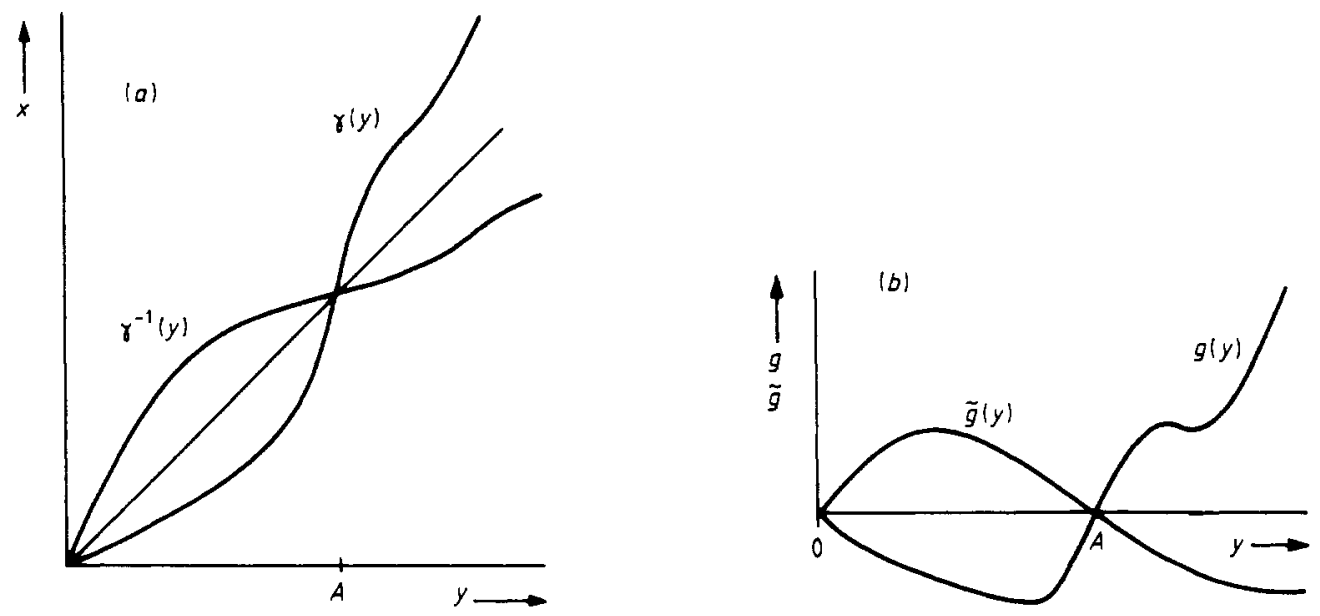

Figure 1. $(a) \gamma^{-1}(y)$ is the mirror image of $\gamma(y)$ with respect to the line $x=y$. (b) Graphs of the functions $g(y)=\gamma(y)-y$ and $\tilde{g}(y)=\gamma^{-1}(y)-y$.

Evaluating $h(y)$ (cf (2.6)) one finds, with expression (3.1) for $A$,

$$
h(y)=2 y(y-A) \text {. }
$$

Clearly $h(y), 0 \leqslant y \leqslant A$, is in $\mathscr{F}_{1}$ and the same holds for $\tilde{\mathrm{g}}$. The latter is defined however only if $\gamma^{\prime}>0$, which is satisfied if $\|g\|<1$. As a result the operator $T$, in (2.6), maps the open set $\{g \mid\|g\|<1\}$ into $\mathscr{F}_{1}$.

Analogously consider the domain $y \geqslant A$, but restrict it to finite values: $y \in[A, D]$ with $D>A$ but arbitrarily large. For this domain the class of functions is

$$
\mathscr{F}_{2} \equiv\left\{g_{2}(y) \mid g_{2} \in \mathscr{C}^{1}([A, D]), g_{2}(A)=0 ; g_{2}^{\prime}(y)>0\right\}
$$

with a distance between two elements

$$
d(u, v)=\sup _{A \leqslant y \leqslant D}\left|u^{\prime}(y)-v^{\prime}(y)\right|, \quad u, v \in \mathscr{F}_{2} .
$$

Note that the extra condition $g^{\prime}>0$ in $(2.11)$ keeps $\mathscr{F}_{2}$ from being a linear space. Since (i) $h(A)=\tilde{g}(A)=0$, (ii) $h^{\prime}(y)>0$ if $y>A$ and (iii) $\tilde{g}^{\prime}<0$ if $g^{\prime}>0$ (cf (2.7), (2.10) and figure 1 ) and since $B$ is non-negative by assumption, $T$ maps $\mathscr{F}_{2}$ into itself. Statement (iii) above follows algebraically from the identity $y=\gamma\left(\gamma^{-1}(y)\right)$, which yields (cf (2.5))

$$
\tilde{g}(y)=-g(y+\tilde{g}(y)) .
$$

After differentiation one obtains

$$
\tilde{g}^{\prime}(y)=-\frac{g^{\prime}(y+\tilde{g}(y))}{1+g^{\prime}(y+\tilde{g}(y))}
$$

which leads to (iii) if $\|g\|<1$.

\section{Existence of the invariant manifold}

Regions in the parameter plane are determined in this section for which the operator $T$ in each case is a contraction. This guarantees unique solutions of the fixed point 
equation as the limit of a sequence $\left\{T^{n} g_{b}\right\}$, with $g_{b}$ arbitrary. Finally error bounds are derived for each step of the iteration.

The fixed points of the Hénon map $(1.1)$ are $(0,0)$ and $(A, A)$ with

$$
A=\frac{1}{2}(1+B-2 C) \text {. }
$$

The characteristic multipliers in each case are

$$
\lambda_{0 \pm}=C \pm\left(C^{2}-B\right)^{1 / 2}, \quad \lambda_{A \pm}=1+B-C \pm\left[(B-C)^{2}+2 A\right]^{1 / 2},
$$

respectively. We confine ourselves to

$$
0<B<1, \quad A>0 \text {. }
$$

The latter inequality implies that $\mathrm{A}$ is a saddle point (cf 3.2)). The range of parameter values for which $T_{1}$ is a contraction mapping is given by (cf figure 2 )

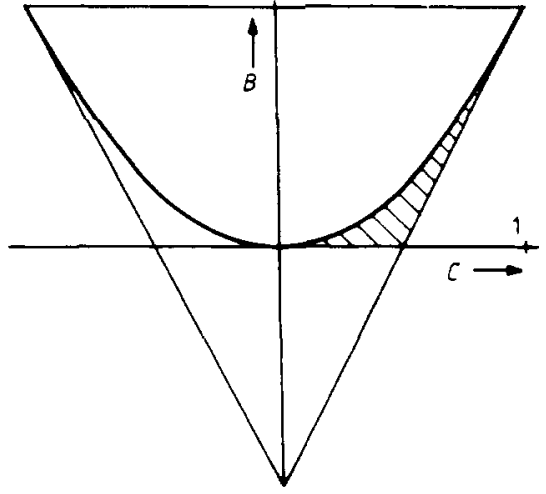

Figure 2. The origin is stable for parameter values in the triangle. Lemma 1 applies for the shaded area.

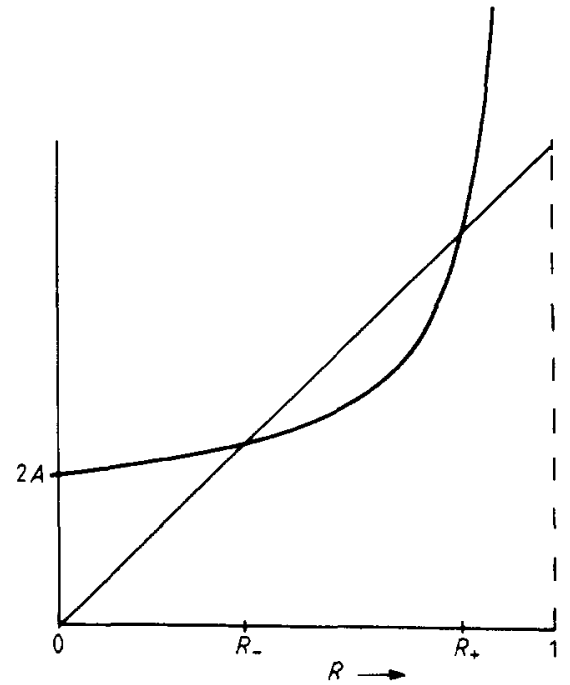

Figure 3. Construction of $R_{+}$and $R_{-}$(cf (3.14) and (3.15)). The curve is the graph of the left-hand side of (3.14).

Lemma 1. For any pair $\{B, C\}$ satisfying (3.3) and

$$
C>0, \quad B<C^{2} \text {, }
$$

there exists an $R, 0<R<1$, and a $\theta, 0<\theta<1$, such that

$$
\begin{aligned}
& \left\|T_{1} g\right\| \leqslant R, g \in \mathscr{F}_{1},\|g\| \leqslant R, \\
& \left\|T_{1} u-T_{1} v\right\| \leqslant \theta\|u-v\|, u, v \in \mathscr{F}_{1},\|u\|,\|v\| \leqslant R .
\end{aligned}
$$

The proof is given at the end of this section. There appears to be some freedom in the actual choice of $R$, and of the corresponding value of $\theta$. One possibility is

$$
R=1-C, \quad \theta=B / C^{2} \text {. }
$$

For an interpretation of the restrictions (3.4), see the remark at the end of $\S 4$.

Analogously we have, for the domain $A \leqslant y \leqslant D$, the following lemma. 
Lemma 2. For any pair $\{B, C\}$ satisfying (3.3) the distance function $d$, defined in (2.12), satisfies

$$
d\left(T_{2} u, T_{2} v\right) \leqslant B d(u, v), \quad u, v \in \mathscr{F}_{2}
$$

Proof. The definition of $d$ yields

$$
\begin{aligned}
d\left(T_{2} u, T_{2} v\right) & =\sup _{A \leqslant y \leqslant D} B\left|\frac{u^{\prime}-v^{\prime}}{\left(1+u^{\prime}\right)\left(1+v^{\prime}\right)}\right| \\
& \leqslant B \sup _{A \leqslant y \leqslant D}\left|u^{\prime}-v^{\prime}\right|=B d(u, v),
\end{aligned}
$$

the inequality being a consequence of the definition of $\mathscr{F}_{2}\left(u^{\prime}\right.$ and $v^{\prime}$ positive).

As $0<B<1, T_{2}$ is a contraction mapping on $\mathscr{F}_{2}$. With these two lemma's one proves in the usual way (cf Liusternik and Sobolev 1965) the following theorem.

\section{Theorem}

(1) If $B$ and $C$ satisfy (3.3) and (3.4) there is in the set $\left\{g \mid g \in \mathscr{F}_{1} ;\|g\| \leqslant R\right\}$ a unique function $g_{1}$ satisfying $g_{1}=T_{1} g_{1}$.

(2) If $B$ and $C$ satisfy (3.3) there is a unique function $g_{2} \in \mathscr{F}_{2}$ satisfying $g_{2}=T_{2} g_{2}$.

With the solutions $g_{1}$ and $g_{2}$ the function $\gamma(y)$ becomes

$$
\begin{aligned}
\gamma(y) & =y+g_{1}(y), & & 0 \leqslant y \leqslant A, \\
& =y+g_{2}(y), & & A \leqslant y<\infty,
\end{aligned}
$$

from which the invariant manifolds $\Gamma_{1}$ and $\Gamma_{2}$ (2.3) are obtained. The upper bound for $y$ in $(3.9 b)$ is ' $\infty$ ', as $D$ is arbitrarily large. In each case the solution is the limit of a sequence $\left\{T^{n} g_{b}\right\}$, where $g_{b}$ is an arbitrary element in the set for which $T$ is a contraction.

Remark. The accuracy with which $T^{n} g_{b}$ approximates the limit is determined easily. Consider the first case. With the aid of (3.5ii) one obtains ( $g_{1}$ denotes a solution)

$$
\left\|T_{1}^{n} g_{b}-g_{1}\right\| \leqslant \theta^{n}\left\|g_{b}-g_{1}\right\| \leqslant \theta^{n}\left(\left\|g_{b}\right\|+R\right), \quad\left\|g_{b}\right\| \leqslant R,
$$

whence, with (2.9) and the inequality $\sup _{0 \leqslant y \leqslant A}|g(y)| \leqslant A \sup _{0 \leqslant y \leqslant A}\left|g^{\prime}(y)\right|$

$$
\sup _{0 \leqslant y \leqslant A}\left|T_{1}^{n} g_{b}-g_{1}\right| \leqslant \theta^{n} A\left(\left\|g_{b}\right\|+R\right)
$$

In the second case similar error bounds could be derived.

Proof of lemma 1. Differentiation of (2.6) yields using (2.14)

$$
\left(T_{1} g(y)\right)^{\prime}=h^{\prime}(y)+B \frac{g^{\prime}(y+\tilde{g}(y))}{1+g^{\prime}(y+\tilde{g}(y))}
$$

and as a result

$$
\left\|T_{1} g\right\| \leqslant\|h\|+B\|g\| /(1-\|g\|)
$$


where $\|h\|=2 A$ (cf (2.10)). To satisfy (3.5i) it is sufficient to have an $R$ such that

$$
2 A+B\|g\| /(1-\|g\|) \leqslant R, \quad 0 \leqslant\|g\|<R .
$$

As $B>0$ the left-hand side is an increasing function of $\|g\|$ and (3.13) is satisfied for any $R$ with

$$
2 A+B \frac{R}{1-R} \leqslant R
$$

Solving this equation for the equality, one finds either two real roots in the interval $[0,1]$, or none, cf figure 3. Equation (3.13) is satisfied only in the first case with $R$ lying between the two roots. From (3.14) one obtains the roots trivially

$$
R_{ \pm}=1-C \pm\left(C^{2}-B\right)^{1 / 2} .
$$

The inequalities (3.4) guarantee that both $R_{+}$and $R_{-}$are in $[0,1]$. To prove (3.5ii) one infers from (3.11)

$$
\begin{aligned}
\|T u-T v\| & \leqslant \frac{B}{(1-\|u\|)} \cdot \frac{1}{(1-\|v\|)}\|u-v\|, \quad\|u\|,\|v\|<1, \\
& \leqslant \frac{B}{(1-R)^{2}}\|u-v\|, \quad\|u\|,\|u\|<R .
\end{aligned}
$$

Taking $R=\frac{1}{2}\left(R_{+}+R_{-}\right)=1-C$ one finds $B /(1-R)^{2}=B / C^{2}$ which is smaller than unity due to (3.4). This proves (3.5ii). Actually one can show that $B /(1-R)^{2}<1$ for any $R$ between $R_{-}$and $R_{+}$.

\section{Discussion}

The invariant manifold considered is shown to be the complete unstable manifold of the saddle. Some characteristic features of its shape are determined. It is pointed out how to use the present method to find an algebraic approximation with known accuracy of the manifold.

The assumptions on $\Gamma$ in $\S 2$ yield the inequalities

$$
\begin{array}{ll}
\gamma(y)<y & \text { if } 0<y<A, \\
\gamma(y)>y & \text { if } y>A .
\end{array}
$$

The second inequality holds by definition, of (2.11). In order to prove the first one recall that the restriction of $H$ to $\Gamma$ is represented by the one-dimensional map $y^{*}=\gamma(y)$. Consequently, since the origin is attracting, the inequality holds for $y$ in an interval $(0, \varepsilon)$. The mapping having only two fixed points, the only solution of $y=\gamma(y)$ are $y=0$ and $y=A$. Hence $(4.1 a)$ follows. These inequalities show that all points on ГO move away monotonically from $A$ under repeated application of the mapping. Therefore $\Gamma O$, is the unstable manifold of $A$. 
Using these results and the construction method of section 3 , we shall now prove

$$
\begin{array}{ll}
\gamma^{\prime}(0)=\lambda_{0+}, & \gamma^{\prime}(A)=\lambda_{A+}, \\
\gamma(y) \leqslant f(y)-B y, & 0 \leqslant y \leqslant A, \\
\gamma(y) \geqslant f(y)-B y, & A \leqslant y, \\
\gamma \text { is smooth; } & \gamma^{\prime \prime}(y)>f^{\prime \prime}(y) .
\end{array}
$$

(i) Since $\Gamma_{1}$ is invariant, $\gamma^{\prime}(0)$ is either equal to $\lambda_{0+}$ or to $\lambda_{0-}$ (cf figure 4). Consequently $g_{1}^{\prime}(0)$ is either equal to $\lambda_{0+}-1$ or to $\lambda_{0-}-1$. The latter possibility however violates the inequality, $\mathrm{cf}(3.2)$ and (3.6),

$$
\left|g^{\prime}(0)\right| \leqslant\|g\| \leqslant R=1-C \text {. }
$$

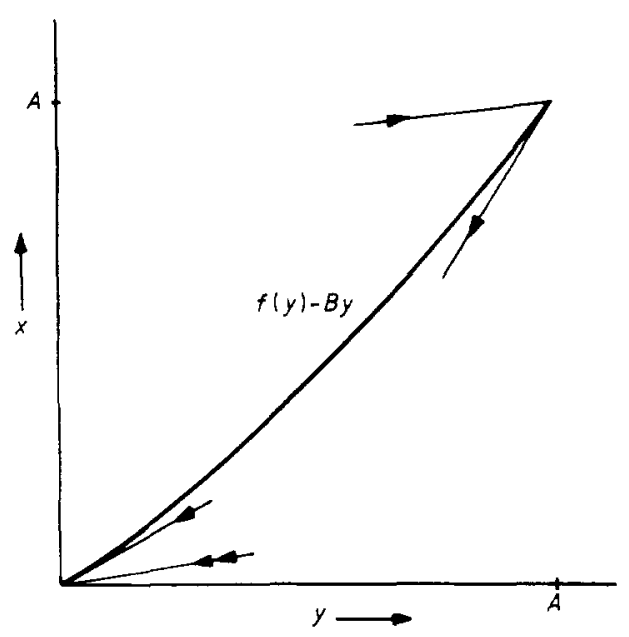

Figure 4. The arrows denote the eigenvectors $\left(\lambda_{0 \pm}, 1\right)$ and $\left(\lambda_{A \pm}, 1\right)$ of the linearisation of $H$ at the fixed points respectively, for $B=0.1$ and $C=0.4$.

The second equality in (i) holds as $\Gamma O$ is the unstable manifold of $A$. (ii) and (iii): The inequalities (4.1) are equivalent with $g_{1}<0$ and $g_{2}>0$ respectively, of (2.5). Hence $\tilde{g}_{1}>0$ and $\tilde{g}_{2}<0$, cf figure 1. Properties (ii) and (iii) then follow from (2.6) and $(2.10)$. In order to prove (iv) note that $H$ depends analytically on $x$ and $y$. Thus the unstable manifold $\Gamma O$ is smooth. The same holds for $\gamma$ and one is allowed to differentiate (2.4) twice to obtain

$$
\gamma^{\prime \prime}(y)=f^{\prime \prime}(y)+\gamma^{\prime \prime}\left(\gamma^{-1}(y)\right) B / \gamma^{\prime 3}\left(\gamma^{-1}(y)\right),
$$

cf (2.6) and (2.13). This yields, after substitution of $y=A$,

$$
\gamma^{\prime \prime}(A)=4 \lambda_{A^{+}}^{3} /\left(\lambda_{A^{+}}^{3}-B\right)
$$

Since $\lambda_{A+}>1$, one obtains $\gamma^{\prime \prime}(A)>4$ in an interval $(-\varepsilon+A, A+\varepsilon)$. Denoting this interval by $\left(y_{0-}, y_{0+}\right)$, one derives from (4.4) that $\gamma^{\prime \prime}(y)>f^{\prime \prime}(y)$ holds in a larger interval $\left(y_{1-}, y_{1+}\right)$, with $y_{1 \pm}=\gamma\left(y_{0 \pm}\right)$. By infinite repetition of this procedure one proves (iv) for $\Gamma \mathrm{O}$.

The method of $\S 3$ can be used to construct an algebraic approximation of known accuracy to the invariant manifold. Consider the initial curve $\Gamma_{1 b}=\left\{\left(x_{0}, y_{0}\right) \mid x_{0}=y_{0}\right.$, 
$0 \leqslant x \leqslant A\}$. This curve is related to its $n$th iterate by $\left(x_{0}, y_{0}\right)=H^{-n}\left(x_{n}, y_{n}\right)$. With the expression for the inverse operator, $H^{-1}(x, y)=\left(y, B^{-1} f(y)-B^{-1} x\right)$, one finds $x_{0}$ and $y_{0}$ in terms of $x_{n}$ and $y_{n}$. Putting $x_{0}=y_{0}$ yields a relation $p_{n}\left(x_{n}, y_{n}\right)=0$, where $p_{n}$ is a polynomial in $x_{n}$ and $y_{n}$. For instance, when $n=1,2$ one obtains

$$
f\left(y_{1}\right)-x_{1}=b y_{1}, \quad f\left(B^{-1} f\left(y_{2}\right)-x_{2}\right)-y_{2}=f\left(y_{2}\right)-x_{2} .
$$

The equation $p_{n}\left(x_{n}, y_{n}\right)=0$ has no unique solution. One branch of the solution however, does give an approximation of $\gamma$, i.e. $x_{n}=\gamma_{n}\left(y_{n}\right)$. Generally an explicit expression for $\gamma_{n}$ cannot be found. It can be solved numerically, however. From (3.10) one sees that $\gamma_{n}$ approximates $\gamma$ to an accuracy better than

$$
\sup _{0 \leqslant y \leqslant A}\left|\gamma_{n}(y)-\gamma(y)\right| \leqslant \theta^{n} R A
$$

where $\theta^{n} R=\left(B / C^{2}\right)^{n}(1-C)$.

Remark. Finally, consider the restrictions (3.4). These are not only sufficient for the present results but also necessary. First, when $B>C^{2}$, the characteristic multipliers of the origin are complex, and an invariant manifold that connects $A$ and $O$ spirals around $O$. Second, if $C<0$ and $B<C^{2}$, the multipliers at $O$ are negative. As a result an invariant manifold must have negative slope at $\mathrm{O}$, which is in contradiction with the a priori assumption (2.1). In order to apply a contraction mapping principle to those cases as well, the invariant manifold should be given a parametrisation different from the present one (cf Francescini and Russo 1981). Work in this direction is in progress.

\section{Acknowledgments}

I thank Robert Helleman for many valuable suggestions. I also acknowledge useful discussions with Reinout Quispel and Johannes van Zeijts, both supported by FOM, and with Barbara Mandl, supported by ZWO. This study was partially supported under DE-AC03-77ER01538.

\section{References}

Bridges R and Rowlands G 1977 Phys. Lett. 63A 189

Brown A L and Page A 1970 Elements of Functional Analysis (London: Van Nostrand Reinhold)

Daido H 1980 Prog. Theor. Phys. 631190

Francescini V and Russo L 1981 J. Stat. Phys. 25757

Helleman R H G 1983 in Long-time Prediction in Dynamics ed C W Horton, L E Reichl and A G Szebehely (New York: Wiley) p 95

Hénon M 1976 Commun. Math. Phys, 5069

Lanford III O E 1983 in Chaotic Behaviour of Deterministic Systems ed G Iooss, R H G Helleman and R Stora (Amsterdam: North-Holland) p 3

Liusternik L A and Sobolev V J 1965 Elements of Functional Analysis (New York: Fred Ungar)

McMillan E M 1971 in Topics in Modern Physics ed W E Brittin and H Odabasi (Boulder, Colorado: Colorado Associated University Press) p 219

Nitecki $Z 1971$ Differentiable Dynamics, An Introduction to the Orbit Structure of Diffeomorphisms (Cambridge, Mass.: MIT Press)

Tel T 1982 Z. Phys. B 49157 